\title{
Rotating vortex imaging implemented by a quantized spiral phase modulation
}

\section{Baránek}

\section{Z. Bouchal}

bouchal@optics.upol.cz
Department of Optics, Palacký University, 17. listopadu 1192/12, 77146 Olomouc, Czech Republic

Department of Optics, Palacký University, 17. listopadu 1192/12, 77146 Olomouc, Czech Republic

We demonstrate both theoretically and experimentally that a spiral mask with a finite number of discrete phase levels allows a defocusing induced rotation of the point spread function in the vortex imaging. Two experimental configurations based on a spiral phase modulation of light and a spiral filtering of the spatial spectrum are studied in a unified theoretical treatment. The rotating point spread functions are analyzed in detail for imaging realized by the vortex lens and the 4 -f system used in the spiral phase contrast microscopy. The theoretical results are verified by experiments using a spatial light modulator. The method is applicable to a precise focusing and optical imaging allowing depth estimation from diffracted rotation. Apart from implementation simplicity, the main advantages of the method are high energy efficiency, a possibility to operate with either complex amplitude or spatial spectrum of light and variability allowing a simple control of number of lobes of the point spread function.

[DOI: http://dx.doi.org/10.2971/jeos.2013.13017]

Keywords: Singular optics, optical vortices, phase modulation, liquid-crystal devices

\section{INTRODUCTION}

Rotating optical beams represent a wide class of light fields whose transversal intensity profile rotates around the axis during propagation in free space [1]-[4]. This feature has been thoroughly investigated theoretically, demonstrated experimentally and utilized in manipulation of atoms and microparticles [5]. Usually, the rotating beams are examined in a Gauss-Laguerre (G-L) modal beam decomposition that enables to find general characteristics of light waves resulting in rotational effects [1]. Methods used in the design of rotating beams can be effectively adapted to the preparation of the double helix point spread function (PSF) in optical microscopy. In this case, the PSF is engineered to have two rotating lobes where the angle of rotation depends on the axial position of the observed point object and significantly increases the sensitivity of depth estimation [6]. In the original design, the rotating PSF system was implemented by a mask placed in the Fourier plane of the 4-f optical system. The mask modulates incident light and generates a superposition of the G-L beams representing the transfer function of the system. The relevant PSF of the system then rotates with defocus. The main disadvantage of this method is its very low transfer function efficiency defined as the ratio of the energy in the main lobes and the energy incident on the mask. Its value is below $2 \%$, so the method is not suitable for photon-limited applications [6]. By using iterative optimization algorithms, the theoretical efficiency of the method can be increased up to $56 \%$. The mask used for the generation of a superposition of the G-L beams then operates without absorption, but the rotational response is available only in a limited area instead of the whole 3D space [7]. The rotating PSF is of great importance for optical microscopy and was used for three-dimensional, single-molecule fluorescence imaging beyond the diffraction limit [8], three-dimensional tracking of microparticles [9], and for the nanoscale localization of pointlike objects [10]. The double-helix PSF was also utilized in holographic optical tweezers enabling parallel manipulation and multiple-particle tracking [11]. Recently, a 3D nanoscopic system based on a Fisher information optimized rotating PSF was proposed and implemented, resulting in the new methodology termed Super-resolution Photon-efficient Imaging by Nanometric Double-helix Localization of Emitters (SPINDLE) [12]. The 3D optical transfer function of rotating beams was also examined and used to increase the axial resolution in the volumetric imaging [13].

In this paper, two different methods of the vortex imaging are proposed and analyzed, in which the rotation of the PSF is achieved by a quantized spiral phase modulation. It is shown that a discrete spiral phase mask with an appropriately chosen number of phase levels produces the PSF with multiple lobes, which rotate when changing the longitudinal position of the observed point object. The image rotation is achieved by interference of the pure vortex modes with different topological charges, which have a full azimuthal overlapping and appear naturally as the Fourier components of a spiral mask with discrete phase levels. Hence, the method has a high efficiency and simple implementation. The discrete spiral phase modulation providing the rotating PSF can be applied in the frequency domain using a 4-f optical system. In contrast to the methods using G-L beams, the discrete spiral phase modulation can also operate in the complex amplitude of light, so that the rotating PSF can be obtained in the lens imaging. This is 
advantageous if the rotating PSF is used for a precise localization of objects in optical microscopy.

The paper is organized as follows. In the second section, a unified mathematical description of the vortex imaging and the 4-f optical system with a spiral filtration is presented. In the third section, the PSF for the vortex imaging with a continuous spiral phase modulation is determined. In the fourth section, the rotation of the PSF is explained as a cooperation of the vortex modes generated by a spiral mask with discrete phase levels. In the fifth section, the rotating PSF is analyzed in detail and conditions of special rotating effects found. In the sixth section, experimental results are demonstrated that verify the correctness of the theoretical analysis of the defocusing induced PSF rotation.

\section{UNIFIED MODEL OF VORTEX IMAGING}

The proposed method of the PSF rotation is based on the vortex imaging, which can be achieved by a spiral phase modulation either in the frequency or spatial domain. If the spiral phase modulation is implemented by the mask whose phase continuously changes with the azimuthal angle, the diffraction image of a point object is changed from the Airy pattern to a doughnut spot. The spiral mask is then characterized by the topological charge $m$ specifying the jump of optical path that occurs when the azimuthal angle is changed by $2 \pi$. We show that the discrete spiral mask with an appropriately chosen number of phase levels creates the vortex modes whose interference results in the defocusing induced rotation of the PSF. A similar mechanism appears also in rotating optical beams where the intensity profile rotates in a free-space propagation. In this paper, the rotational effects are studied in a unified mathematical description usable for both the vortex lens imaging and the spiral filtering implemented by the 4-f optical system. First, a mathematical description of a continuous spiral phase modulation will be proposed and subsequently effects caused by the discrete spiral modulation will be thoroughly investigated.

\subsection{Vortex lens with spiral phase modulation}

The vortex imaging with a continuous spiral modulation is investigated for the geometry shown in Figure 1. Monochromatic radiation with the wavelength $\lambda$ is emitted by a point source and transformed by the vortex lens, whose operation is specified by the aperture function $G$, the quadratic phase associated with the lens focal length $f$, and the spiral phase function $S$ corresponding to a continuous spiral phase mask with the topological charge $m$.

The complex amplitude describing imaging of a point object is then given by the Fourier transform

$$
\begin{aligned}
\psi\left(\mathbf{r}_{\perp}^{\prime}\right) \propto \iint_{-\infty}^{\infty} & G\left(\mathbf{r}_{\perp}\right) S\left(\mathbf{r}_{\perp}\right) \exp \left[i \frac{k \Delta \Phi}{2}\left|\mathbf{r}_{\perp}\right|^{2}\right] \\
& \times \exp \left[i 2 \pi \frac{\mathbf{r}_{\perp} \cdot \mathbf{r}_{\perp}^{\prime}}{\lambda z^{\prime}}\right] d \mathbf{r}_{\perp},
\end{aligned}
$$

where $k=2 \pi / \lambda, \mathbf{r}_{\perp} \equiv(x, y), \mathbf{r}_{\perp}^{\prime} \equiv\left(x^{\prime}, y^{\prime}\right), z=z_{0}+\Delta z$ and

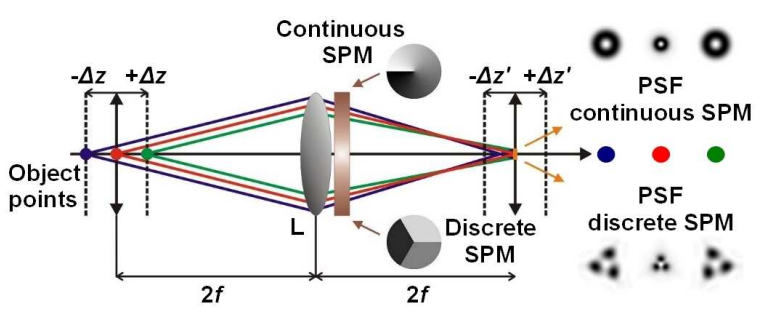

FIG. 1 Schema of vortex imaging with the rotating PSF.

$z^{\prime}=z_{0}^{\prime}+\Delta z^{\prime} . \Delta \Phi$ describes the image defocusing and can be written as

$$
\Delta \Phi=\frac{1}{f}-\frac{1}{z}-\frac{1}{z^{\prime}} \approx \frac{\Delta z}{z_{0}^{2}}+\frac{\Delta z^{\prime}}{z_{0}^{\prime 2}} .
$$

The image is sharp only when the point source and the detection plane are at the positions $z_{0}$ and $z_{0}^{\prime}$ fulfilling the lens equation. If the detection plane is fixed at the distance $z_{0}^{\prime}$, the shift of a point source to the position $z_{0}+\Delta z$ causes the image defocusing. A similar situation arises when the source is in the position $z_{0}$ and the detection plane is shifted to the distance $z_{0}^{\prime}+\Delta z^{\prime}$. As will be shown later, the defocusing shifts $\Delta z$ and $\Delta z^{\prime}$ can be sensitively indicated by the PSF rotation when a spiral phase mask with discrete phase levels is used as a phase modulating element.

\subsection{Optical 4-f system with spiral filtering}

The PSF rotation is also examined for the 4-f optical system, which performs a spiral phase modulation of the spatial spectrum. This system has been successfully used in spiral phase contrast microscopy and its principle is explained in Figure 2. The light wave generated by a point source placed near the object focal plane is transformed by the lens $L_{1}$ and modulated by the spiral phase filter placed in the image focal plane. A lateral bounding of the wave is given by the aperture function of the lens $G$ and can be also applied at the image focal plane of the lens. This simplification is acceptable because the wave is nearly collimated.

The helical light wave is then transformed by the lens $L_{2}$ and a doughnut image spot is detected near the image focal plane. The optical path between object and image planes can be written in terms of the Fresnel diffraction theory. The complex amplitude of the image can then be expressed as

$$
\begin{aligned}
\psi\left(\mathbf{r}_{\perp}^{\prime}\right) \propto \iint_{-\infty}^{\infty} & G\left(\mathbf{r}_{\perp}\right) S\left(\mathbf{r}_{\perp}\right) \exp \left[-i \frac{k\left|\mathbf{r}_{\perp}\right|^{2}}{2}\left(\frac{1}{Z}+\frac{1}{f_{2}}\right)\right] \\
& \times \iint_{-\infty}^{\infty} \exp \left(-i \frac{k\left|\mathbf{R}_{\perp}\right|^{2}}{2 z^{\prime}}\right) \\
& \times \exp \left[i k \mathbf{R}_{\perp} \cdot\left(\frac{\mathbf{r}_{\perp}}{f_{2}}+\frac{\mathbf{r}_{\perp}^{\prime}}{z^{\prime}}\right)\right] d \mathbf{R}_{\perp} d \mathbf{r}_{\perp}
\end{aligned}
$$

where $\mathbf{R}_{\perp} \equiv(X, Y), Z=-f_{1}^{2} / \Delta z$ and $z^{\prime}=f_{2}+\Delta z^{\prime}$.

The second integral is the Fourier transform of a quadratic phase function and can be calculated analytically. The com- 


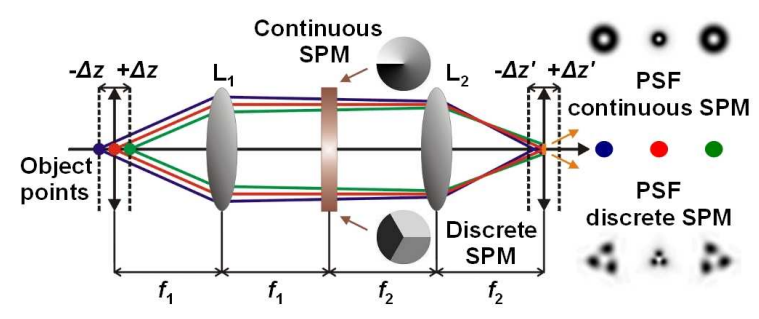

FIG. 2 Illustration of the PSF rotation in the 4 -f optical system.

plex amplitude then can be simplified to the form

$$
\begin{aligned}
\psi\left(\mathbf{r}_{\perp}^{\prime}\right) \propto \iint_{-\infty}^{\infty} & G\left(\mathbf{r}_{\perp}\right) S\left(\mathbf{r}_{\perp}\right) \exp \left[i \frac{k \Delta \Phi}{2}\left|\mathbf{r}_{\perp}\right|^{2}\right] \\
& \times \exp \left[i 2 \pi \frac{\mathbf{r}_{\perp} \cdot \mathbf{r}_{\perp}^{\prime}}{\lambda f_{2}}\right] d \mathbf{r}_{\perp},
\end{aligned}
$$

where $G$ and $S$ again denote the aperture and spiral modulation functions but this time expressed in the coordinates of the image focal plane of the lens $L_{1}$, and $\Delta \Phi$ is a defocusing term. It can be written as

$$
\Delta \Phi=\frac{z^{\prime}}{f_{2}^{2}}-\frac{1}{f_{2}}-\frac{1}{Z}=\frac{\Delta z}{f_{1}^{2}}+\frac{\Delta z^{\prime}}{f_{2}^{2}} .
$$

As is obvious, the point image is described by formally identical relations in Eqs. (1) and (4) valid for both the vortex lens imaging, and the spiral filtration in the 4-f system. The only difference is the form of the function $\Delta \Phi$ that describes the image defocusing. The unified mathematical formalism simplifies the analysis of the PSF rotation in both practically important cases of the vortex imaging.

\section{Vortex imaging with continuous spiral phase modulation}

To solve the integrals in Eqs. (1) and (4), it is appropriate to introduce the cylindrical coordinates $r, \varphi$ given as $x=r \cos \varphi$ and $y=r \sin \varphi$. In a similar way, the image space coordinates $r^{\prime}, \varphi^{\prime}$ are used. The function $S$ corresponding to the spiral mask with a continuous azimuthal change of the phase can then be written as

$$
S=\exp (i m \varphi)
$$

where $m$ is an integer indicating the topological charge of the mask. In optical imaging, the aperture function corresponding to a circular aperture is usually used. Here, we use a Gaussian apodization given by $G=\exp \left(-\left|\mathbf{r}_{\perp}\right|^{2} / \Delta r^{2}\right)$, where $\Delta r$ is the commonly defined radius of the Gaussian profile. This approach simplifies the calculations and does not affect the analysis of the rotation of the PSF, which is the main objective of the paper. Under these assumptions, the integrals in Eqs. (1) and (4) can be solved analytically. The complex amplitude describing the image of a point object can then be expressed as

$$
\begin{aligned}
\psi\left(r^{\prime}, \varphi^{\prime}\right) \propto & i^{m} \frac{v^{\prime}}{g^{3 / 2}} \exp \left(i m \varphi^{\prime}-\frac{\pi^{2} v^{\prime 2}}{2 g}\right) \\
& \times\left[I_{\frac{1}{2}(m-1)}\left(\frac{\pi^{2} v^{\prime 2}}{2 g}\right)-I_{\frac{1}{2}(m+1)}\left(\frac{\pi^{2} v^{\prime 2}}{2 g}\right)\right],
\end{aligned}
$$

where $I_{n}$ denotes the modified Bessel function of the $n$-th order and

$$
g=\frac{1}{\Delta r^{2}}-i \frac{k}{2} \Delta \Phi
$$

Eq. (7) is valid only if the order of the Bessel functions satisfies the inequality $n>-2$ [14]. It is applicable to both configurations of the vortex imaging, but $\Delta \Phi$ and $v^{\prime}$ differ in individual cases,

$$
\Delta \Phi=\frac{\Delta z}{z_{0}^{2}}+\frac{\Delta z^{\prime}}{z_{0}^{\prime 2}}, \quad v^{\prime}=\frac{r^{\prime}}{\lambda z^{\prime}} \cdots \text { for vortex lens, }
$$

$$
\Delta \Phi=\frac{\Delta z}{f_{1}^{2}}+\frac{\Delta z^{\prime}}{f_{2}^{2}}, \quad v^{\prime}=\frac{r^{\prime}}{\lambda f_{2}} \cdots \text { for spiral filtering. }
$$

As can be seen, the light field in the image space has a helical wavefront defined by $\exp \left(i m \varphi^{\prime}\right)$. The pitch of the helix is specified by the topological charge of the used mask $m$. If the spiral mask is not continuous and has a finite number of discrete phase levels, the light field consists of helical modes with different topological charges. Under appropriate conditions, their interference leads to the rotation effects.

\section{Vortex imaging with quantized spiral phase modulation}

In experiments, the spiral mask is implemented by a spatial light modulator (SLM) or by photolithography techniques and its phase is sampled to a finite number of levels. If the number of phase levels of the discrete mask is sufficiently high, it operates as a continuous mask in a very good approximation. In the case of the mask with a small number of phase levels, the PSF becomes rotationally asymmetrical and its rotation occurs when the image is out of focus. To quantify the dependence of the PSF rotation on defocus shifts, a calculation model is proposed including effects caused by the spiral phase sampling. We consider a discrete mask composed of $L$ angular segments that cause constant phase changes. The phase of the $l$-th segment is given as

$S_{l}=\exp [i m 2 \pi(l-1) / L]$ for $\varphi \in[2 \pi(l-1) / L, 2 \pi l / L]$,

where $m$ is the intrinsic topological charge of the mask, $L$ is the total number of phase levels and $l=1,2, \cdots, L$, denotes the sequence number of the phase segments. An action of the mask is then given by the function $S=\sum_{l} S_{l}$. Because the mask is periodic in $\varphi$, it can be written using the Fourier series,

$$
S(\varphi)=\sum_{n=-\infty}^{+\infty} c_{n} \exp (\operatorname{in} \varphi) .
$$

If the transparency of the mask is known, the Fourier coefficients can be obtained by

$$
c_{n}=\frac{1}{2 \pi} \int_{0}^{2 \pi} S(\varphi) \exp (-i n \varphi) d \varphi
$$

When the mask with the segments given by Eq. (11) is inserted into Eq. (13), its Fourier coefficients can be calculated and arranged to the form [15],

$$
c_{n}= \begin{cases}\exp \left(i \frac{n \pi}{L}\right) \operatorname{sinc}\left(\frac{n}{L}\right), & n=m-s L, s=0, \pm 1, \pm 2, \ldots \\ 0, & \text { others. }\end{cases}
$$


To facilitate calculations, it is appropriate to adjust Eq. (12) so that the summation is performed only over the positive indices. If $S$ is then substituted into Eqs. (1) and (4) and the Fourier transform is calculated, we get

$$
\begin{aligned}
\psi\left(r^{\prime}, \varphi^{\prime}\right) \propto & \frac{v^{\prime}}{g^{3 / 2}} \exp \left(-\frac{\pi^{2} v^{\prime 2}}{2 g}\right) \\
& \times \sum_{n=1}^{\infty} i^{n}\left[c_{-n} \exp \left(-i n \varphi^{\prime}\right)+c_{n} \exp \left(i n \varphi^{\prime}\right)\right] \\
& \times\left[I_{\frac{1}{2}(n-1)}\left(\frac{\pi^{2} v^{\prime 2}}{2 g}\right)-I_{\frac{1}{2}(n+1)}\left(\frac{\pi^{2} v^{\prime 2}}{2 g}\right)\right],
\end{aligned}
$$

where $g, \Delta \Phi$ and $v^{\prime}$ are given by Eqs. (8)-(10). The intensity $I=|\psi|^{2}$ normalized to a unit maximum then represents the PSF for the spiral imaging implemented by a quantized spiral phase modulation. Unlike the case with a continuous spiral mask, the PSF does not have rotational symmetry and the intensity profile depends on the azimuthal angle $\varphi^{\prime}$.

\section{Rotation of out of focus image}

The image rotation can be studied using Eq. (15) derived under conditions that include both defocusing and quantized spiral modulation. The rotational effects are first proved by approximate analytical calculations and subsequently analyzed numerically and demonstrated experimentally.

\subsection{Approximate analysis of image rotation}

The calculation of the PSF can be simplified by the fact that a quantized spiral mask may be represented in a good approximation by only two dominant members of the Fourier series. As can be shown by numerical evaluation of Eq. (14), the spiral phase mask with the intrinsic topological charge $m$ and the number of phase levels $L$ is represented with sufficient accuracy by terms with coefficients $c_{m}$ and $c_{(m-L)}$. In the case of defocusing, $g$ is a complex function and the modified Bessel functions used in Eq. (15) are also complex. In subsequent analysis they will be denoted as

$$
I_{m}\left(\frac{\pi^{2} v^{\prime 2}}{2 g}\right)-I_{n}\left(\frac{\pi^{2} v^{\prime 2}}{2 g}\right) \equiv A_{m, n} \exp \left(i \Omega_{m, n}\right) .
$$

The intensity I corresponding to the PSF can then be written as

$$
\begin{aligned}
& I\left(r^{\prime}, \varphi^{\prime}\right) \propto \\
& \quad v^{\prime 2} I_{0}\left[C_{m}^{2} A_{\frac{1}{2} m_{-}, \frac{1}{2} m_{+}}^{2}+C_{m-L}^{2} A_{\frac{1}{2}\left(L-m_{+}\right), \frac{1}{2}\left(L-m_{-}\right)}^{2}\right] \\
& \quad+v^{\prime 2} I_{0} i^{2 m+3 L} C_{m} C_{m-L} A_{\frac{1}{2} m_{-}, \frac{1}{2} m_{+}} A_{\frac{1}{2}\left(L-m_{+}\right), \frac{1}{2}\left(L-m_{-}\right)} \\
& \quad \times\left\{e^{i\left(L \varphi^{\prime}+\Delta \Omega+\pi\right)}+(-1)^{L} e^{-i\left(L \varphi^{\prime}+\Delta \Omega+\pi\right]}\right\},
\end{aligned}
$$

where

$$
\begin{aligned}
I_{0} & =\frac{1}{|g|^{3}} \exp \left[-\frac{\pi^{2} v^{\prime 2} \Re\{g\}}{|g|^{2}}\right], \\
\Delta \Omega & =\Omega_{\frac{1}{2} m_{-}, \frac{1}{2} m_{+}}-\Omega_{\frac{1}{2}\left(L-m_{+}\right), \frac{1}{2}\left(L-m_{-}\right)^{\prime}} \\
C_{n} & =\operatorname{sinc}\left(\frac{n}{L}\right), \quad n=m, L-m,
\end{aligned}
$$

and the used indices indicate $m_{ \pm}=m \pm 1$. The image of a point source is now expressed as an interference law for two general vortex fields with topological charges $M_{1}$ and $M_{2}$ fulfilling the condition $M_{1}-M_{2}= \pm L$. The investigated mask with $L$ phase levels and the intrinsic topological charge $m$ is represented by $M_{1}=m$ and $M_{2}=m-L$.

\subsubsection{Defocusing-induced image rotation}

The detailed analysis of (17) demonstrates that the intensity profile can rotate during image defocusing. The conditions leading to the rotation are investigated separately for two different cases in which the number of phase levels $L$ is given by an odd or even number. As the simplest example, the imaging implemented by a mask with the intrinsic topological charge $m=1$ and the number of phase levels $L=3$ is discussed. In this case, the intensity can be simplified to the form

$$
\begin{aligned}
I\left(r^{\prime}, \varphi^{\prime}\right) \propto & v^{\prime 2} I_{0}\left[C_{1}^{2} A_{0,1}^{2}+C_{-2}^{2} A_{\frac{1}{2}, \frac{3}{2}}^{2}\right. \\
& \left.+2 C_{1} C_{-2} A_{0,1} A_{\frac{1}{2}, \frac{3}{2}} \sin \left(3 \varphi^{\prime}+\Delta \Omega+\pi\right)\right]
\end{aligned}
$$

where $\Delta \Omega=\Omega_{0,1}-\Omega_{\frac{1}{2}, \frac{3}{2}}$. The lateral intensity distribution of the image spot is bounded by the Gaussian function $I_{0}$ whose width depends on the function $g$ describing the defocusing. As a result, the image spot spreads if the out of focus shift $\Delta z$ or $\Delta z^{\prime}$ increases. If the image is in focus, $g$ given by Eq. (8) becomes real and the modified Bessel functions $I_{n}$ are also real functions. The phases $\Omega_{n}$ are then equal to zero for an arbitrary index $n$ and $\Delta \Omega=0$. At the plane of best focus, the image spot has three lobes with the intensity maxima in the directions specified by the angles $\varphi_{\max }^{\prime}=\pi / 2,7 \pi / 6$ and $11 \pi / 6$. If the image is out of focus then $\Delta \Omega \neq 0$, and the intensity maxima appear in the directions given by the angles $\varphi_{\max }^{\text {out }}=\varphi_{\max }^{\prime}-\Delta \Omega / 3$. With regard to Eq. (16), $\Delta \Omega$ depends on $g$ and thus also on $\Delta z$ and $\Delta z^{\prime}$. The dependence of $\varphi_{\text {max }}^{\text {out }}$ on the out of focus shifts indicates that the intensity spot rotates during the defocusing. The specific form of the dependence of the rotation angle on the defocusing shift was found by an approximate analysis based on the properties of the Bessel functions and by numerical simulations. It was verified that the dependence of $\varphi_{\max }^{\text {out }}$ on $\Delta z$ reminds the inverse tangent function with the slope of the linear region depending on the numerical aperture of the used optical system. This theoretical prediction is in excellent accordance with the experimental results.

\subsubsection{Rotation invariant imaging}

The imaging implemented by a quantized spiral mask can remain rotation invariant during defocusing only if the intrinsic topological charge and the number of phase levels are suitably adapted. To demonstrate this case, the spiral mask with the intrinsic topological charge $m$ and the number of phase levels $L=2 m$ must be used. With these parameters, $m_{-}=L-m_{+}$ and $m_{+}=L-m_{-}$, so that $\Delta \Omega=0$ for an arbitrary defocusing $\Delta z$ or $\Delta z^{\prime}$, and the image spot does not rotate. As the simplest example, the imaging implemented by the quantized spiral mask with $m=1$ and $L=2$ can be demonstrated,

$$
I \propto v^{\prime 2} I_{0} C_{1}^{2} A_{0,1}^{2} \sin ^{2} \varphi^{\prime} .
$$

The image spot is formed by two lobes that spread during defocusing but do not rotate. According to Eq. (17), the number 
of lobes is uniquely determined by the number of phase levels $L$ and does not depend on the intrinsic topological charge of the mask $m$. This allows to create both the rotating and nonrotating images with the same number of lobes.

\subsubsection{Images with reverse rotation}

The rotation of the PSF depends on the phase difference $\Delta \Phi=\Phi_{i, j}-\Phi_{p, s}$, where the indices $i, j$ and $p, s$ are defined by Eq. (17) as $i=m_{-} / 2, j=m_{+} / 2, p=\left(L-m_{+}\right) / 2$ and $s=\left(L-m_{-}\right) / 2$. A simple analysis shows, that the images created by the quantized phase masks with $L$ phase levels and the intrinsic topological charges $m_{1}$ and $m_{2}$, rotate in reverse directions, if the condition $L=m_{1}+m_{2}$ is satisfied. The reverse rotation appears as a consequence of the mutual exchange of indices $i \leftrightarrow p$ and $j \leftrightarrow s$ resulting in $\Delta \Phi_{1}=\Delta \Phi$ and $\Delta \Phi_{2}=-\Delta \Phi$.

In Figure 3, the reverse rotation is demonstrated for three- and four-level spiral phase masks. In the left panel, in focus and

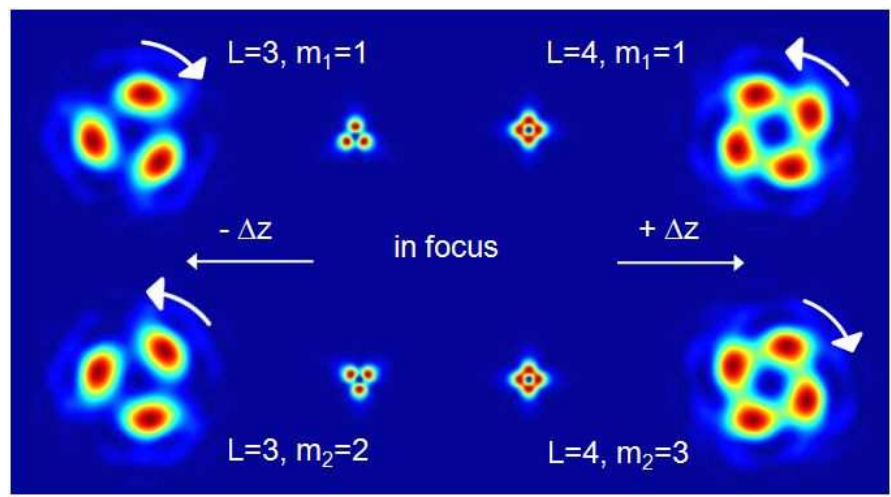

FIG. 3 Demonstration of the reverse image rotation obtained for the phase masks with the topological charges $m_{1}$ and $m_{2}$, whose number of phase levels is given by $L=m_{1}+m_{2}$. Left panel: in focus and out of focus PSF for a negative defocusing and parameters $L=3, m_{1}=1$ and $m_{2}=2$, respectively. Right panel: the same as in left panel but for a positive defocusing and $L=4, m_{1}=1$ and $m_{2}=3$, respectively.

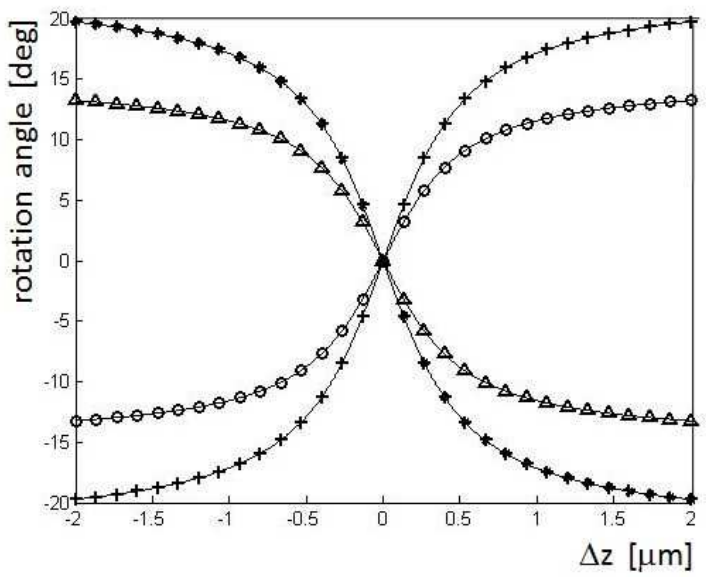

FIG. 4 Dependence of the PSF rotation on the defocusing shift $\Delta z$. The reverse rotations are demonstrated for the spiral masks whose number of phase levels $L$ and the intrinsic topological charges $m_{1}$ and $m_{2}$ satisfy the condition $L=m_{1}+m_{2}$ : '000' $\ldots$ $L=3, m_{1}=1,{ }^{\prime} \Delta \Delta \Delta^{\prime} \cdots L=3, m_{2}=2,{ }^{\prime}+++{ }^{\prime} \cdots L=4, m_{1}=1$ and ' $* * *$ ' $L=4, m_{2}=3$. out of focus PSF is compared for $L=3$ and the intrinsic topological charges $m_{1}=1$ and $m_{2}=2$, respectively. As is demonstrated, the images rotate in the reverse directions under the same negative defocusing. In the right panel, the reverse image rotation is shown for the mask with $L=4$ and the intrinsic topological charges $m_{1}=1$ and $m_{2}=3$, respectively. The dependence of the PSF rotation on the defocusing is illustrated in Figure 4 for three- and four-level masks. The curves showing the exact reverse rotation are again obtained for the masks with the intrinsic topological charges adapted to the number of phase levels, $L=m_{1}+m_{2}$. As is evident, the maximal achievable rotation increases with an increasing number of phase levels $L$.

\section{Experimental results and comparison with theory}

Although the PSF rotation was verified successfully for both the vortex imaging and the spiral filtration, in this paper only the results obtained in the 4-f system illustrated in Figure 5 are presented. This set-up is commonly used in spiral phase contrast microscopy $[16,17]$, in which the edge contrast enhancement of phase objects is achieved by means of a continuous spiral phase mask placed in the plane, where the spatial spectrum of the specimen is created. The method proposed in this paper allows applying the same system also for the longitudinal location of pointlike objects by means of the rotating PSF. To achieve this operation, the only required modification of the system is the replacement of the continuous spiral mask by a discrete mask with a finite number of phase levels.

In the verification experiment, the He-Ne laser coupled into a single-mode fiber was used as a point source. Light emanating from the fiber was collimated by the microscope objective MO (Melles Griot, NA=0.55, $50 \times$ ) and transferred on an active display of the SLM (Boulder, $512 \times 512$ ) using a relay optics composed of the lenses $L_{1}$ and $L_{2}$. The spiral mask with a quantized phase ensuring the PSF rotation was displayed on the SLM together with the grating providing separation of modulated light from the zero diffraction order. The SLM was placed in the back focal plane of the lens $L_{2}$ and the light reflected in the first diffraction order was captured by the lens $L_{3}$ realizing the inverse Fourier transform. The rotating PSF was detected by the CCD in the focal plane of the lens $L_{3}$.

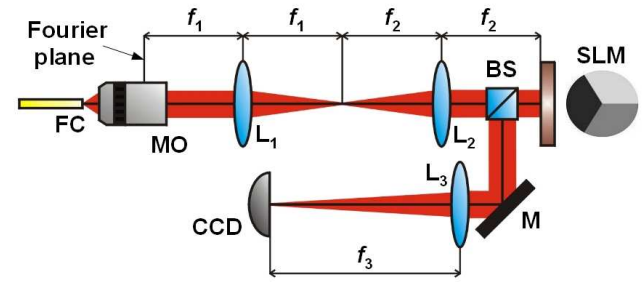

FIG. 5 Experimental verification of the PSF rotation implemented by a quantized spiral phase modulation in the $4-f$ system: $\mathrm{FC}$ - optical fiber $\mathrm{NA}=0.1$ (He-Ne laser, $20 \mathrm{~mW}$, $632.8 \mathrm{~nm}$ ), MO - microscope objective (Melles Griot NA=0.55, 50X), SLM - spatial light modulator (Boulder, 512×512), BS - beam splitter, $M$ - mirror, $L_{1}, L_{2}, L_{3}$ - lenses ( $f_{1}=f_{2}=200 \mathrm{~mm}, f_{3}=400 \mathrm{~mm}$ ), CCD - Olympus, F-View II. 


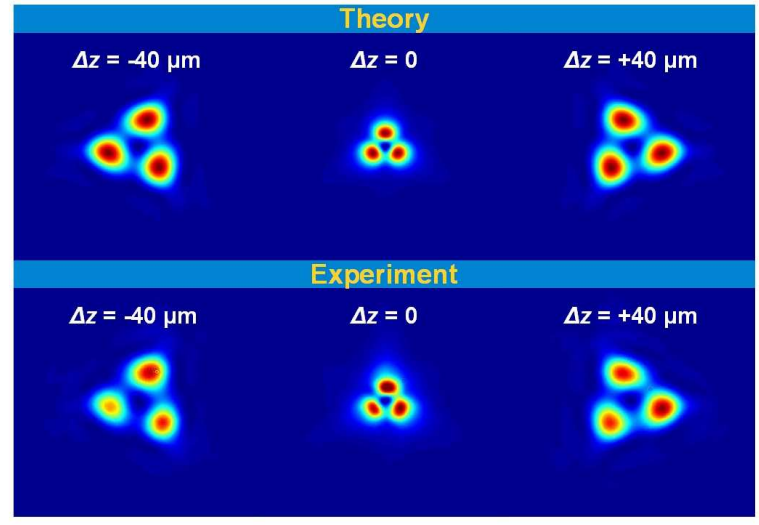

FIG. 6 Numerical simulations (top row) and experimental verification (bottom row) of a defocusing-induced rotation of the PSF in the 4 - $f$ system with the discrete spiral phase mask (object-side numerical aperture $N A=0.1$, intrinsic topological charge $m=1$, number of phase levels $L=3$, defocusing shift $\Delta z= \pm 40 \mu \mathrm{m}, \Delta z^{\prime}=0$ ).

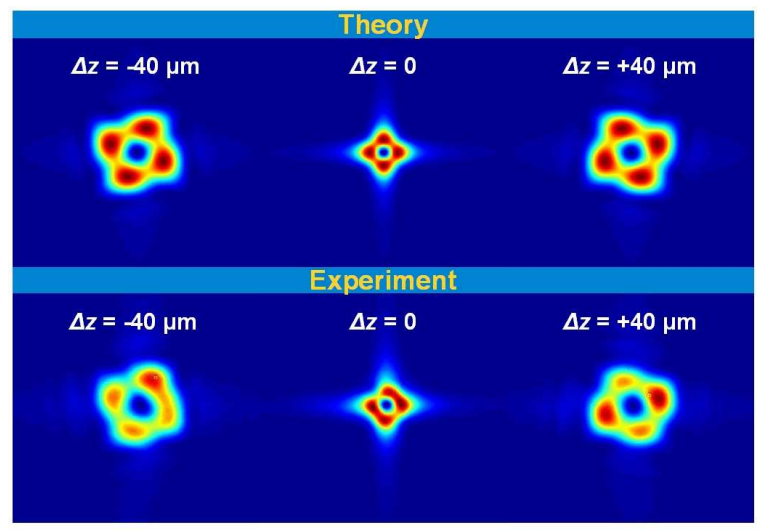

FIG. 7 The same as in Figure 6, but for the topological charge $m=1$ and the number of phase levels $L=4$.

The correctness of the theoretical model proposed for the evaluation of the rotating imaging was experimentally verified for special combinations of the intrinsic topological charge $m$ of the spiral phase mask and the number of phase levels $L$. In Figures 6 and 7, the theoretical and experimental results demonstrating the defocusing-induced rotation of the PSF are compared for a quantized spiral phase mask with the parameters $m=1$ and $L=3$ and $L=4$, respectively. The theoretical results (top row) were obtained by numerical simulations using Eq. (15), the experimental verification (bottom row) was carried out in the set-up shown in Figure 5. The object-side numerical aperture of the microscope objective MO was limited by the output numerical aperture of the used optical fiber $(N A=0.1)$. Defocusing was performed in the object space by a longitudinal shift of the fiber $(\Delta z= \pm 40 \mu \mathrm{m})$, the CCD was located at the focal plane of the lens $L_{3}\left(\Delta z^{\prime}=0\right)$. In accordance with Eq. (17), the number of lobes of the PSF is directly determined by the number of phase levels of the mask $L$. As was previously demonstrated by Eq. (18), the mask with the intrinsic topological charge $m$ and the number of phase levels $L$ creates a pair of dominant vortex fields with the topological charges $m$ and $L-m$, whose interference causes the rotation of the defocused image. In cases demonstrated in Figures 6 and 7, the PSF expands and rotates clockwise or anticlock-

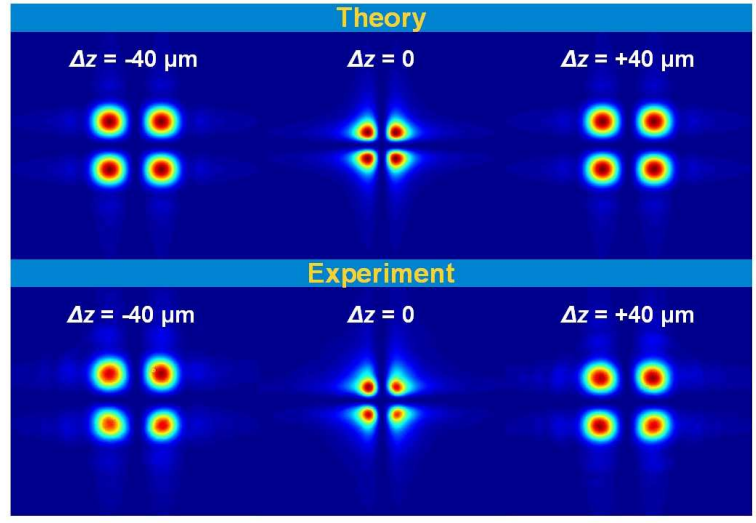

FIG. 8 The same as in Figure 6, but for the topological charge $m=2$ and the number of phase levels $L=4$. In accordance with the theoretical analysis, the PSF does not rotate during defocusing, because the condition $L=2 m$ is satisfied.

wise depending on the direction of the defocusing shift. On the contrary, the results of the approximate rotation analysis indicate that the PSF remains rotationally invariant during defocusing when the condition $L=2 m$ is fulfilled. This feature allows to create both the rotating and non-rotating PSF with the same number of lobes. As an illustrative example, the PSF generated by the mask with $m=2$ and $L=4$ is used. Rotating and non-rotating versions of the PSF with four lobes are demonstrated in Figures 7 and 8 with a very good agreement between theory and experiment.

To use the PSF rotation for a localization of pointlike objects, dependence of the rotation angle on the defocusing shift must be determined. This task can be solved using analytical or numerical calculation methods. As is obvious from Eq. (17), the PSF rotates by the angle $\Delta \varphi^{\prime o u t}=\Delta \Omega / L$, if the object or detector are shifted outside the object or image paraxial plane. Using the established symbols, $\Delta \Omega$ can be written as

$$
\Delta \Omega=\arctan \frac{\Im\left(I_{p}-I_{q}\right)}{\Re\left(I_{p}-I_{q}\right)}-\arctan \frac{\Im\left(I_{r}-I_{S}\right)}{\Re\left(I_{r}-I_{S}\right)} .
$$

For spiral mask with the parameters $m$ and $L$, the indices become $p=m_{-} / 2, q=m_{+} / 2, r=\left(L-m_{+}\right) / 2$ and $s=$ $\left(L-m_{-}\right) / 2$. The dependence of $\Delta \Omega$ on the defocusing shifts $\Delta z$ and $\Delta z^{\prime}$ is intricately hidden in the complex argument of the Bessel functions Eq. (16). A detailed analysis can be done using the properties of the Bessel functions. Numerical analysis shows that the dependence of the rotation angle on the defocusing shift is similar to the arctangent function. To determine the position of pointlike objects, the linear part is used, whose slope varies with the numerical aperture of optics used. Numerical and experimental results are demonstrated in Figure 9. The numerical analysis was performed for the spiral mask with $m=1$ and $L=3$ in the 4-f set-up using lenses with the numerical apertures $N A=0.1,0.3$ and 0.6 , respectively. As is obvious, the PSF rotation is more sensitive to defocusing at higher numerical apertures $N A$, and also the maximal achievable rotation angle of the intensity profile is greater when $N A$ is increased. Experimental results obtained for $N A=0.1$ closely match the simulation. 


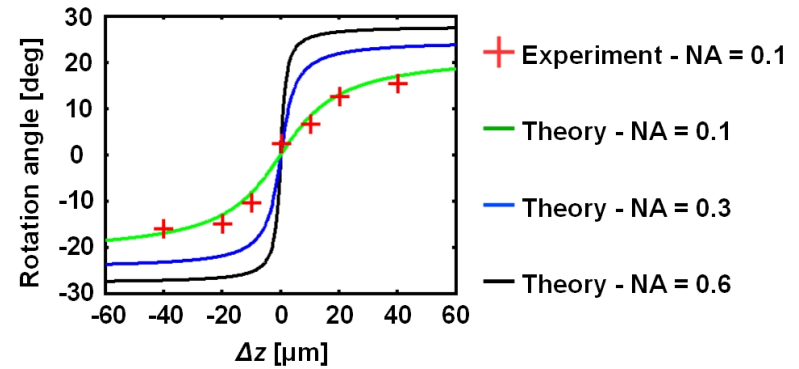

FIG. 9 Theoretical and experimental dependence of the PSF rotation on the object-side defocusing $\Delta z$ for the spiral mask with $m=1$ and $L=3$ and different numerical apertures of the microscope objective MO.

\section{CONCLUSION}

In the paper, the rotating vortex imaging is examined in two basic experimental configurations based on a quantized spiral phase modulation. The studied configurations represent either a vortex lens, where the spiral modulation is applied directly to the complex amplitude of light, or the 4-f system, which allows the spiral modulation of the spatial spectrum. Analysis of both systems is based on a unified mathematical description enabling determination of the PSF. The main attention is devoted to the rotating effects that occur when the spiral modulation with a quantized phase is used and the image is out of focus. The main theoretical and experimental results can be summarized as follows:

- The PSF valid for both experimental configurations of the vortex imaging was determined and applied to analysis of the defocused image created by the spiral modulation with a quantized phase. It was shown that the image of a point object is given by an infinite sum of the vortex fields with different topological charges (Eq. (15)). In this treatment, the quality of the spiral imaging using a quasicontinuous mask with a large number of phase levels can be assessed.

- It was verified that the spiral imaging implemented by the spiral mask with the intrinsic topological charge $m$ and the low number of phase levels $L$ can be described by the PSF determined by a superposition of two dominant vortex modes. Their topological charges depend on the mask parameters and are given as $m$ and $L-m$, respectively. An approximate two-mode analysis provides a sufficient accuracy and was used to clarify the reasons for the image rotation when the spiral modulation with a quantized phase is applied.

- A detailed analysis of the PSF rotation caused by the defocusing was performed and special rotational regimes found. When the spiral mask with $L$ phase levels is used, the PSF has $L$ lobes and rotates during defocusing. The rotation is due to interference of the vortex modes generated by the mask and depends on its intrinsic topological charge $m$ and the number of phase levels $L$. It was verified both theoretically and experimentally that the PSF remains rotationally invariant only if $L=2 m$, in all other cases the PSF rotates during defocusing.
- Using the proposed theoretical models, the condition of the reverse PSF rotations was found and experimentally verified in the 4 -f system. It was shown that two point images can rotate in the exactly reverse directions even if the same defocusing shift was applied. The reverse rotation occurs, when the images were created by the spiral masks with $L$ phase levels and the topological charges $m_{1}$ and $m_{2}$, which satisfy the condition $L=m_{1}+m_{2}$. This property was used in the correlation method enabling determination of the angular rotation of the PSF from the detected intensity spots.

- It was shown that the dependence of the rotation angle of the PSF on the defocusing shift corresponds to the arctangent function. The slope of the linear region usable for applications increases with increasing numerical aperture of optics used and the number of phase levels of the spiral mask. The usable range of the angular rotation can be adjusted using the mask parameters.

The defocusing rotation of the image can be used as a sensitive tool for the precise localization of pointlike objects. In previous experiments, the image rotation was implemented in optical systems with the double helix PSF [6]-[8],[10]. The PSF rotation proposed in the paper is based on the quantized spiral phase modulation which can be applied either to the spatial spectrum or the complex amplitude of light. This is advantageous for microscopy because the particle localization does not require the Fourier filtering in the 4-f system and can be realized by a direct lens imaging.

\section{ACKNOWLEDGEMENTS}

This work was supported by the Technology Agency of the Czech Republic, project No. TE01020229 and the IGA project of the Palacký University PrF 2012-05.

\section{References}

[1] Y. Y. Schechner, R. Piestun, and J. Shamir, "Wave propagation with rotating intensity distributions," Phys. Rev. E 54, R50-R53 (1996).

[2] V. V. Kotlyar, V. A. Soifer, and S. N. Khonina, "An algorithm for the generation of laser beams with longitudinal periodicity: rotating images," J. Mod. Opt. 44, 1409-1416 (1997).

[3] S. N. Khonina, V. V. Kotlyar, V. A. Soifer, M. Honkanen, J. Lautanen, and J. Turunen, "Generation of rotating Gauss-Laguerre modes with binary-phase diffractive optics," J. Mod. Opt. 46, 227-238 (1999).

[4] A. Y. Bekshaev, M. S. Soskin, and M. V. Vasnetsov, "Angular momentum of a rotating light beam," Opt. Comm. 249, 367-378 (2005).

[5] M. P. MacDonald, L. Paterson, K. Volke-Sepulveda, J. Arlt, W. Sibbett, and K. Dholakia, "Creation and manipulation of threedimensional optically trapped structures," Science 296, 1101-1103 (2002).

[6] A. Greengard, Y. Y. Schechner, and R. Piestun, "Depth from diffracted rotation," Opt. Lett. 31, 181-183 (2006).

[7] S. R. P. Pavani and R. Piestun, "High-efficiency rotating point spread functions," Opt. Express 16, 3484-3489 (2008). 
[8] S. R. P. Pavani, M. A. Thompson, J. S. Biteen, S. J. Lord, N. Liu, R. J. Twieg, R. Piestun, and W. E. Moerner, "Threedimensional, single-molecule fluorescence imaging beyond the diffraction limit by using a double-helix point spread function," PNAS 106, 2995-2999 (2009).

[9] S. R. P. Pavani and R. Piestun, "Three dimensional tracking of fluorescent microparticles using a photon-limited double-helix response system," Opt. Express 16, 22048-22057 (2008).

[10] M. D. Lew, S. F. Lee, M. Badieirostami, and W. E. Moerner, "Corkscrew point spread function for far-field three-dimensional nanoscale localization of pointlike objects," Opt. Lett. 36, 202-204 (2011).

[11] D. B. Conkey, R. P. Trivedi, S. R. P. Pavani, I. I. Smalyukh, and R. Piestun, "Three-dimensional parallel particle manipulation and tracking by integrating holographic optical tweezers and engineered point spread functions," Opt. Express 19, 3835-3842 (2011).

[12] G. Grover, K. DeLuca, S. Quirin, J. Deluca, and R. Piestun, "Superresolution photon-efficient imaging by nanometric double-helix point spread function localization of emitters (SPINDLE)," Opt. Express 20, 26681-26695 (2012).
[13] M. R. Hatzvi and Y. Y. Schechner, "Three-dimensional optical transfer of rotating beams," Opt. Lett. 37, 3207-3209 (2012).

[14] M. Abramowitz and I. A. Stegun, Handbook of mathematical functions (U.S. Government Printing office, Washington, D.C., 1972).

[15] Ch. S. Guo, D. M. Xue, and Y. J. Han, "Optimal phase steps of multilevel spiral phase plates," Opt. Commun. 268, 235-239 (2006).

[16] S. Furhapter, A. Jesacher, S. Bernet, and M. Ritsch-Marte, "Spiral phase contrast imaging in microscopy," Opt. Express 13, 689-694 (2005).

[17] A. Jesacher, S. Furhapter, S. Bernet, and M. Ritsch-Marte, "Shadow effects in spiral phase contrast microscopy," Phys. Rev. Lett. 94, 233902 (2005). 